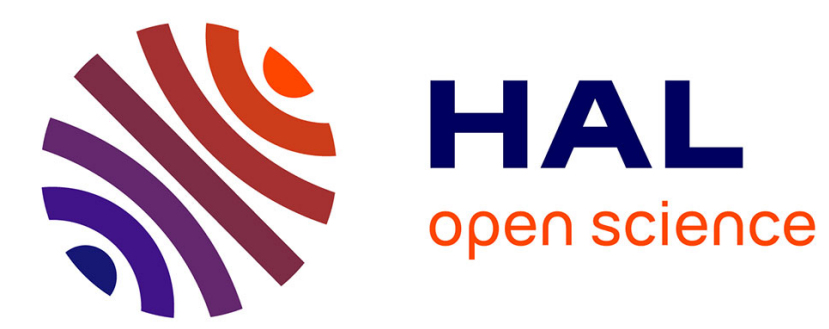

\title{
Falling styles of disks
}

Franck Auguste, Jacques Magnaudet, David Fabre

\section{To cite this version:}

Franck Auguste, Jacques Magnaudet, David Fabre. Falling styles of disks. Journal of Fluid Mechanics, 2013, vol. 719, pp.388-405. 10.1017/jfm.2012.602 . hal-00908124

\section{HAL Id: hal-00908124 https://hal.science/hal-00908124}

Submitted on 22 Nov 2013

HAL is a multi-disciplinary open access archive for the deposit and dissemination of scientific research documents, whether they are published or not. The documents may come from teaching and research institutions in France or abroad, or from public or private research centers.
L'archive ouverte pluridisciplinaire HAL, est destinée au dépôt et à la diffusion de documents scientifiques de niveau recherche, publiés ou non, émanant des établissements d'enseignement et de recherche français ou étrangers, des laboratoires publics ou privés. 


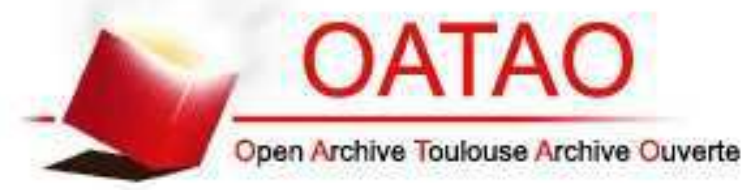

This is an author-deposited version published in : http://oatao.univ-toulouse.fr/ Eprints ID : 10246

To link to this article : DOI:10.1017/jfm.2012.602

URL : http://dx.doi.org/10.1017/jfm.2012.602

To cite this version :

Auguste, Franck and Magnaudet, Jacques and Fabre, David Falling styles of disks. (2013) Journal of Fluid Mechanics, vol. 719 .pp. 388-405. ISSN 0022-1120

Any correspondence concerning this service should be sent to the repository administrator: staff-oatao@ listes.diff.inp-toulouse.fr 


\title{
Falling styles of disks
}

\author{
Franck Auguste $^{1}$, Jacques Magnaudet ${ }^{1,2, \dagger}$ and David Fabre ${ }^{1}$ \\ ${ }^{1}$ Université de Toulouse, INPT, UPS, IMFT (Institut de Mécanique des Fluides de Toulouse), \\ Allée Camille Soula, F-31400 Toulouse, France \\ ${ }^{2}$ CNRS, IMFT, F-31400 Toulouse, France
}

We numerically investigate the dynamics of thin disks falling under gravity in a viscous fluid medium at rest at infinity. Varying independently the density and thickness of the disk reveals the influence of the disk aspect ratio which, contrary to previous belief, is found to be highly significant as it may completely change the route to non-vertical paths as well as the boundaries between the various path regimes. The transition from the straight vertical path to the planar fluttering regime is found to exhibit complex dynamics: a bistable behaviour of the system is detected within some parameter range and several intermediate regimes are observed in which, although the wake is unstable, the path barely deviates from vertical. By varying independently the body-to-fluid inertia ratio and the relative magnitude of inertial and viscous effects over a significant range, we set up a comprehensive map of the corresponding styles of path followed by an infinitely thin disk. We observe the four types of planar regimes already reported in experiments but also identify two additional fully three-dimensional regimes in which the body experiences a slow horizontal precession superimposed onto zigzagging or tumbling motions.

Key words: bifurcation, flow-structure interactions, nonlinear dynamical systems

\section{Introduction}

The variety of paths exhibited by paper strips, dead leaves or winged seeds falling down to the ground has attracted attention of scientists for a long time (see Ern et al. $(\square)$ for a brief review). It is now widely recognized that, although such bodies may be shown mathematically to display non-straight motions even in an inviscid fluid (Lamb $\square$ ), vorticity resulting from the no-slip condition at the body surface plays a crucial role in generating the time-dependent forces and torques that actually control the characteristics of the observed paths. Axisymmetric bodies such as ellipsoids and disks are among the simplest three-dimensional bodies for which the interplay of the body geometrical anisotropy and wake-induced loads can be studied in detail to better understand why, how and when the path of a freely falling (or rising) body deviates from vertical.

Several experimental investigations with falling disks of various thicknesses have been carried out over the last 50 years (Willmarth, Hawk \& Harvey $\square$; Stringham, Simons \& Guy $\square$; Field et al. $\square$; Fernandes et al. $\square$ ), some of which 
include qualitative visualizations of the wake (Willmarth et al. $\square$; Fernandes et al. $\square$ ). By varying the disk density and fluid viscosity, the solid-to-fluid inertia ratio and the ratio between inertial and viscous forces were varied independently, allowing regime maps based on the path geometry to be established. Four main regimes were identified: when viscous effects are large enough, the disk falls broadside along a straight vertical path. Then, as inertial effects increase, three non-vertical but planar types of path were observed, depending on the solid-to-fluid inertia ratio. The disk may display a zigzagging (or fluttering) motion during which it performs incomplete rotations about a horizontal axis while falling along a vertical mean path. When the disk inertia increases, the rotations may become complete, yielding the autorotation (or tumbling) regime in which the disk centre of mass follows an inclined path. These two periodic states are separated by an intermediate chaotic regime consisting of successive, randomly distributed, periods of zigzags and autorotation. In what follows these four planar regimes are referred to as SV (straight vertical), ZZ (zigzag), AR (autorotation) and ZZ/AR (zigzag/autorotation), respectively. An additional threedimensional regime in which the disk spirals along a vertical axis without drifting horizontally has recently been identified with disks of very low inertia (Zhong, Chen \& Lee $\square$ ).

\section{Problem definition and computational strategy}

We use direct numerical simulation to explore the dynamics of disks over a wide range of solid-to-fluid inertia ratios in regimes where viscous effects, although small, are still significant. The disk is considered to have a homogeneous density $\rho_{s}$, diameter $d$ and thickness $h$. It falls within an infinite body of Newtonian fluid of density $\rho$ and kinematic viscosity $v$ at rest at infinity. The problem depends on three control parameters, namely the disk aspect ratio $\chi=d / h$, the body-to-fluid density ratio $\bar{\rho}=\rho_{s} / \rho$ (most often replaced by the dimensionless inertia ratio $I^{*}=(\pi / 64) \bar{\rho} / \chi$ (Willmarth et al. $\square$ ) and the Archimedes number $A r=\sqrt{(3 / 32)} U_{g} d / v$ which is a Reynolds number based on the gravitational velocity $U_{g}=\{2|\bar{\rho}-1| g h\}^{1 / 2}$. For a disk of given mass, the definitions of $I^{*}$ and $A r$ remain meaningful as $\chi \rightarrow \infty$ provided the finite surface density $\sigma_{s}=\rho_{s} h$ is introduced, yielding $I^{*}=(\pi / 64) \sigma_{s} / \rho d$ and $U_{g}=\left(2 g \sigma_{s}|1-(1 / \bar{\rho})| / \rho\right)^{1 / 2}$. It is also frequently useful to refer to a Reynolds number $R e_{m}=U_{m} d / \nu$ based on the average vertical velocity $U_{m}$ measured throughout the disk descent; however, although $R e_{m}$ has frequently been used to establish experimental configuration maps, it is not a true control parameter of the problem since the disk velocity is not known a priori. Periodic paths and disk oscillations of frequency $f$ are characterized by a reduced frequency or Strouhal number $S t=f d / U_{g}$ and a maximum inclination of the disk with respect to the vertical $\theta$.

In what follows we explore the dynamics of disks with $\chi \geqslant 10$ in the range $1.5 \times 10^{-3} \leqslant I^{*} \leqslant 0.5$ and $12 \leqslant A r \leqslant 110$, which approximately corresponds to $25 \leqslant R e_{m} \leqslant 300$. Available experiments have covered a much wider range of Reynolds number $\left(20 \leqslant R e_{m} \leqslant 3 \times 10^{4}\right.$, approximately), but most of them focused on the region $3 \times 10^{-3} \leqslant I^{*} \leqslant 6 \times 10^{-2}, 30 \leqslant R e_{m} \leqslant 2 \times 10^{3}$, where all four aforementioned planar regimes were encountered.

The general strategy we employ to solve the fluid + body problem was extensively described by Mougin \& Magnaudet $(\square,\ulcorner)$ and will not be repeated here. In brief, the Navier-Stokes equations written in a reference frame fixed in the laboratory but with axes rotating with the disk are integrated in time by keeping the translational and rotational disk velocities fixed during each time step. Then these velocities are 
updated by solving the proper form of the generalized Kirchhoff-Kelvin equations expressing Newton's second law for a rigid body moving in an incompressible viscous fluid (Mougin \& Magnaudet $\square$ ). In particular, the time rate of change of the body translational and rotational velocities is evaluated by considering the inertia of the complete fluid + solid system, so that no convergence issue is encountered with bodies of small or even zero proper inertia. The fluid problem is spatially discretized on a large cylindrical boundary-fitted grid moving with the disk. Details regarding boundary conditions, grid characteristics and accuracy tests are provided in the appendix A. In what follows, the instability of the system is triggered by initially imposing a small disturbance either on the disk inclination or on its rotation rate. When the final regime is reached for a given pair of parameters $\left(I^{*}, A r\right)$, the corresponding solution is used as an initial condition to examine the behaviour at both slightly higher and slightly lower $A r$.

\section{What does 'thin' mean for a disk?}

In experimental investigations, the disks necessarily have a finite thickness; varying this thickness is routinely used to obtain variations of $I^{*}$ with a given material. Willmarth et al. ( $\square$ ), Stringham et al. ( $\square$ ), Field et al. ( $\square$ ) and Zhong et al. ( $\square$ ) employed disks with $\chi \geqslant 10$ (most of which in the range $10 \leqslant \chi \leqslant 60$ ), assuming implicitly that, for a given value of $I^{*}$, the dynamics observed with such large aspect ratios do not differ significantly from those corresponding to $\chi \rightarrow \infty$. We revisited this assumption by examining the $\mathrm{SV}-\mathrm{ZZ}$ transition sequence for disks with a fairly small inertia $\left(4 \times 10^{-3} \leqslant I^{*} \leqslant 5 \times 10^{-2}\right)$ and various aspect ratios. In particular, we examined the behaviour of two disks corresponding to $\chi=10$ and $\chi=\infty$, respectively.

Figure $\Gamma$ compares the behaviour of two disks with a similar relative inertia $I^{*} \approx 4 \times 10^{-3}$. With an infinitely thin disk (figure $\lceil a-b$ ), the $\mathrm{ZZ}$ regime can be observed within the range $33 \leqslant A r \leqslant 46$. Throughout this range, this path is characterized by variations of the instantaneous disk velocity by at least a factor of two (as depicted by the green area in figure $\ulcorner a$ ), significant maximum inclinations $\theta$ ranging from $23^{\circ}$ to $35^{\circ}$ (figure $\ulcorner b$ ), as well as large lateral displacements $l_{h}\left(0.5 d \lesssim l_{h} \lesssim 0.7 d\right)$. The Strouhal number in this regime is $S t \approx 0.235$ at the onset and weakly increases with $A r$. For $A r \geqslant 46$, the system switches to a fully three-dimensional $\mathrm{HH}$ (hula-hoop) regime which is basically a zigzagging regime in which the plane of the successive zigzags slowly precesses about the vertical axis (see §4.2). This regime is observed at least up to $A r=55$ where we stopped the computations. In this range, the characteristics of this new regime remain almost constant $\left(S t \approx 0.25 ; \theta \approx 35^{\circ} ; l_{h} \approx 0.7 d\right)$. The sharp onset of the $\mathrm{ZZ}$ regime at $A r=33$ suggests that the $\mathrm{SV}-\mathrm{ZZ}$ transition is subcritical. Indeed, starting from lower values of $A r$, we found that the SV path remains stable beyond this threshold, up to $A r_{S V}=34.3\left(R e_{m} \approx 90\right)$. Furthermore, increasing $A r$ again, a series of regimes characterized by tiny deviations of the path from the vertical and small disk inclinations $\left(\theta<2^{\circ}\right)$ are observed. These regimes, which are detected up to $A r \approx 43$, are collectively termed $A$-regimes hereinafter following the nomenclature of Ern et al. ( $\square$ ). Starting from $A r=34.3$ and increasing $A r$, one successively observes: (i) a spiral path of extremely small amplitude $\left(\approx 10^{-5} d\right)$ with a Strouhal number $S t \approx 0.20$; (ii) a spiralling motion with an elliptical horizontal projection 

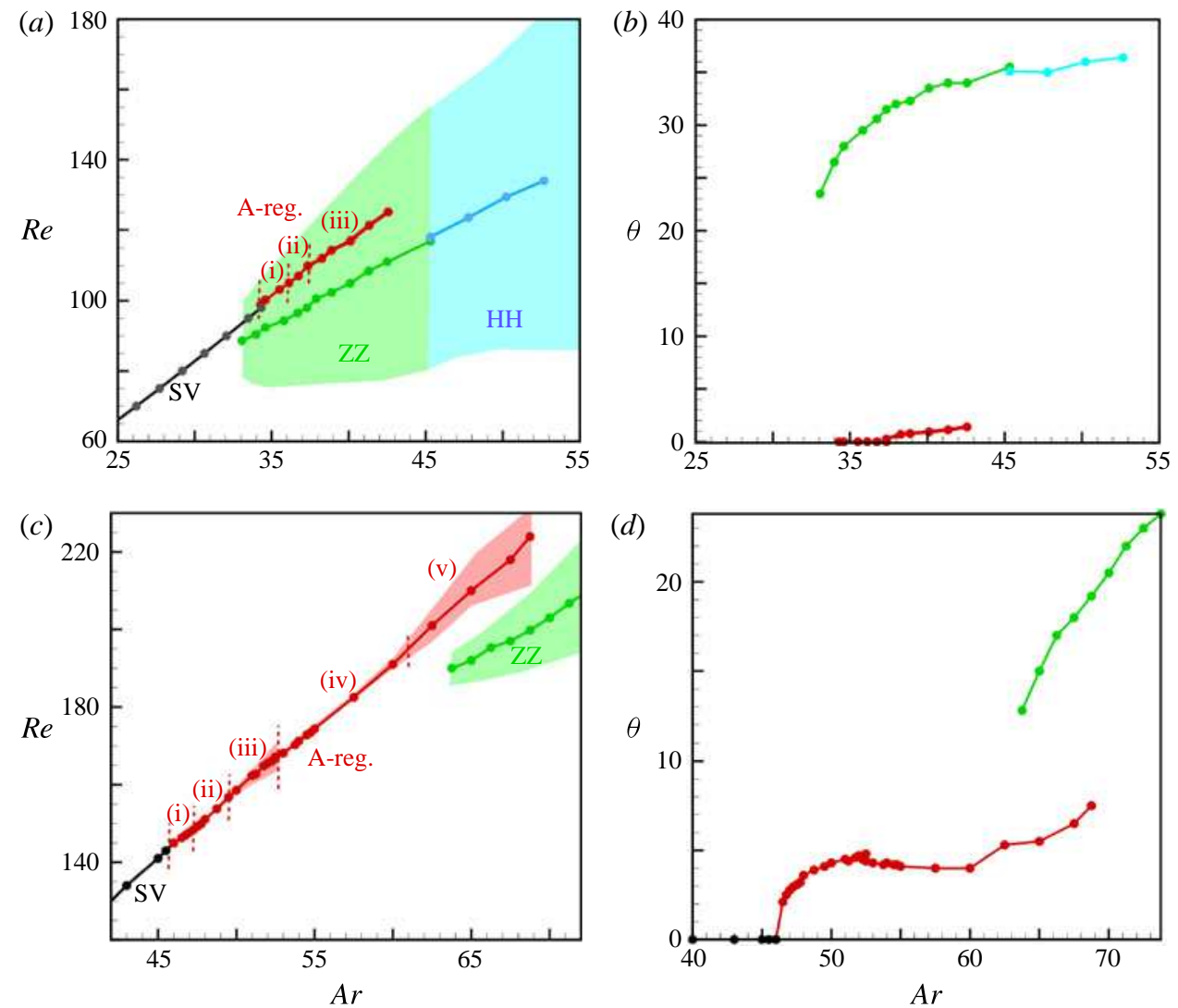

FIGURE 1. Reynolds number $R e$ and maximum inclination $\theta$ versus the Archimedes number Ar for two disks with $I^{*} \approx 4 \times 10^{-3}$ : (a) $\operatorname{Re}$ and $(b) \theta$ for $\chi=\infty ;(c) \operatorname{Re}$ and $(d) \theta$ for $\chi=10$. In $(a, c)$ the lines and superimposed symbols correspond to the mean Reynolds number based on the average falling velocity, while the shaded areas represent the region spanned by the instantaneous Reynolds number for regimes in which a significant deviation from the mean is observed.

having a major axis $\sim 10^{-2} d$; and (iii) a planar periodic path which differs from the previously described $\mathrm{ZZ}$ path by a much lower amplitude $\left(\sim 10^{-1} d\right)$ and a lower frequency $(S t \approx 0.08)$.

In the case of a disk with $\chi=10$ (figure $\ulcorner c-d$ ), the SV-ZZ transition is also found to be subcritical. The $\mathrm{ZZ}$ regime is observed down to $A r \approx 63.5\left(\operatorname{Re}_{m} \approx 190\right)$, and at least up to $A r=110\left(\operatorname{Re}_{m} \approx 295\right)$ where the computations were stopped. The Strouhal number is $\approx 0.205$ at the onset and the variations of the instantaneous velocity are significant, albeit more limited than with $\chi=\infty$. Starting from lower values of $A r$, the SV regime is found to be stable up to $A r_{S V}=46$, and is succeeded by a series of five low-amplitude $A$-regimes which are detected up to $A r \approx 68.5$. The first three of them are planar and correspond respectively to: (i) a steady oblique path, the existence of which was recently confirmed using a totally different approach (Fabre, Tchoufag \& Magnaudet $\square$ ); (ii) a periodic regime with tiny oscillations about a mean oblique path; and (iii) a quasi-periodic regime resembling the previous one but involving a low-frequency modulation. The last two $A$-regimes are three-dimensional 
and correspond respectively to; (iv) a helical path of very large pitch with small radial modulations; and (v) a chaotic regime.

In the various $A$-regimes encountered with both disks, the linear relation linking the time-averaged Reynolds number $\langle R e\rangle$ and the Archimedes number (solid lines in figure $\Gamma a, c$ ) throughout the SV regime remains nearly unchanged. In the $\mathrm{ZZ}$ and $\mathrm{HH}$ regimes, another quasilinear $\langle R e\rangle-A r$ relation with a significantly lower slope emerges, indicating that the disk falls more slowly than would be predicted by extrapolating the former linear relation. The reason for this change of slope is two-fold. First, as soon as the disk starts oscillating, part of the potential energy of the whole system is spent to 'feed' its horizontal motion rather than its fall. Second, compared to the axisymmetric wake associated with the SV path, wakes associated with non-straight paths involve vortical structures with larger velocity gradients (as will be seen in $\S 5.2$ ), resulting in a larger viscous dissipation, i.e. a larger drag of the disk (Fabre et al. $\square$ ).

To summarize, the above two cases share a subcritical transition to the $\mathrm{ZZ}$ path, a significant range of bistability, and the existence of a series of low-amplitude $A$ regimes. However, they strongly differ by three main aspects. First, the characteristic Archimedes numbers corresponding to the destabilization of the SV regime and even more to the onset of the $\mathrm{ZZ}$ regime are substantially larger for $\chi=10$ than for $\chi=\infty$. Second, the two series of $A$-regimes are completely different, as is the order in which the various symmetries of the system are broken or recovered. Last but not least, the range of bistability is much larger for $\chi=\infty$, the ZZ path then coexisting with the $A$-regimes throughout their range of existence and even with the SV regime within a small range of $A r$. Similar differences between disks with $\chi=\infty$ and $\chi=10$ were observed with values of $I^{*}$ typically one order of magnitude higher than those of the above two cases. For instance, with $I^{*} \approx 4.9 \times 10^{-2}$, we found the threshold of the $\mathrm{ZZ}$ regime to be $A r \approx 15.5$ for an infinitely thin disk and $A r \approx 41$ for a disk with $\chi=10$. Additional computations with $\chi=25$ and $\chi=50$ also revealed that, for a given $I^{*}$, the threshold $A r_{S V}$ and the characteristics of the $\mathrm{ZZ}$ regime still significantly depend on $\chi$ for such large aspect ratios. These findings seriously question available $\left(I^{*}, R e_{m}\right)$ regime maps gathering experimental results obtained with disks of very different aspect ratios. Based on present results, especially on the large variation of the thresholds we noticed, the frontiers between the four regimes pictured in these maps must be regarded as essentially qualitative (note however that our computations for disks with finite $\chi$ were restricted to the range $4 \times 10^{-3} \leqslant I^{*} \leqslant 5 \times 10^{-2}$ and most of them concentrated on the SV-ZZ transition. Therefore we cannot provide conclusions about the influence of the aspect ratio on higher-order transitions, except for $I^{*} \approx 4 \times 10^{-3}$ where present results show that a disk with $\chi=10$ does not experience any secondary transition at least up to $A r=110$, in contrast with the infinitely disk which switches from a planar $\mathrm{ZZ}$ path to a three-dimensional $\mathrm{HH}$ path for $A r=46)$. Moreover, the $\mathrm{SV}-\mathrm{ZZ}$ transition does not reduce to a single curve in the $\left(I^{*}, A r\right)$ plane, even for $\chi=\infty$, since a region of bistability and a large variety of intermediate $A$-regimes in which the path only barely deviates from the vertical are found to exist. Such tiny deviations are frequently reported in experiments but can hardly be characterized because the accuracy of the measurement systems is generally not sufficient to resolve them properly and also because it is extremely hard to get rid of small spurious fluctuating motions in the carrying fluid (Fernandes et al. $\square$; Ern et al. $)$.

Several mechanisms contribute to make the dynamics of thin disks with a given $I^{*}$ still significantly dependent on their aspect ratio for $\chi \geqslant 10$. First, the vorticity production at the edge of the disk gets more intense as $\chi \rightarrow \infty$, contributing to make 
the wake intrinsically more unstable, as can be checked by computing the steady flow past a disk held fixed at normal incidence in a uniform stream: while the wake becomes unstable at $R e \approx 115.5$ when $\chi=\infty$, the threshold is delayed until $R e \approx 127$ when $\chi=10$ (Auguste $\square$ ). Also, the drag on a disk translating edgewise at a given Reynolds number goes on decreasing with $\chi$ at large aspect ratio (for $R e=100$, it is $\sim 20 \%$ larger for $\chi=10$ than for $\chi=\infty$ ), especially because the pressure contribution to that drag component is entirely supported by the lateral surface and only slowly fades away as $\chi \rightarrow \infty$. Then, the translational and rotational added-mass coefficients of the disk are decreasing functions of $\chi$; one vanishes when $\chi \rightarrow \infty$ while the other two decrease by more than $15 \%$ from $\chi=10$ to $\chi=\infty$ (Loewenberg $\square$ ). In contrast, the proper inertia of the disk does not vary significantly with $\chi$ in that range. Therefore the total (i.e. fluid+solid) inertia opposing the disk translational or rotational accelerations is significantly less with $\chi=\infty$ than with $\chi=10$. The cumulated effect of these variations makes the disk motion more unstable and facilitates its edgewise translations as $\chi \rightarrow \infty$, keeping the dynamics of the system $\chi$-dependent even for aspect ratios of several tens. We did not try to determine beyond which aspect ratio the behaviour of the disk can be considered to be $\chi$-independent. Nevertheless, we may point out that a weakly nonlinear analysis carried out with a distinct code (Fabre et al. $\square$ ) recently revealed that the bifurcation from the SV state to the first non-vertical steady path (encountered above for $\chi=10$ in the (i)-regime of figure $\Gamma c$ ) switches from supercritical to subcritical for $\chi \approx 52$, confirming that structural changes still take place in the dynamics of the system at such large aspect ratios. The recent linear stability analysis of Assemat, Fabre \& Magnaudet ( $\square$ ) also provides evidence of a qualitatively similar influence of $\chi$ on the instability threshold of low-inertia two-dimensional falling cards.

We believe that the sensitivity to the aspect ratio exhibited by thin disks is generic of thin bodies and not primarily related to the presence of sharp corners bounding the edge. The key physical reason we see for this influence is the fact that flow disturbances induced by the relative acceleration between the body and fluid have to turn by $180^{\circ}$ around the edge: the thinner the body, the larger the curvature imposed by this change of direction to the associated streamlines, irrespective of the precise shape of the edge. Our view is reinforced by a recent computational study (Chrust $\square$ ) in which freely moving oblate spheroids with various inertia and aspect ratios were considered. This study revealed for instance that, for $I^{*}=6.25 \times 10^{-3}$, the SV regime extends up to significantly larger Archimedes numbers for $\chi=6\left(A r_{S V} \approx 44\right)$ than for $\chi=\infty\left(A r_{S V} \approx 33\right)$, and that the steady oblique and oscillating oblique $A$-regimes are present in the former case but not in the latter. These findings are clearly in line with those reported in figure $\ulcorner$ for disks, although no sharp corner is involved in the body geometry.

\section{Styles of fall}

Figure $\ulcorner$ gathers the various well-defined fall regimes observed within the whole set of computations carried out for $A r<55$ with infinitely thin disks. A supplementary movie of each regime is available at http://dx.doi.org/10.1017/jfm.2012.602. In addition to the four classical planar regimes identified by Field et al. ( $\square)$, the present map reveals existence of two additional non-planar regimes which have not been documented before, which we term as hula-hoop $(\mathrm{H})$ and helical autorotation (HA), respectively. Both of them are characterized by a slow precession of the plane within which the disk falls while zigzagging in the former case or tumbling in the 


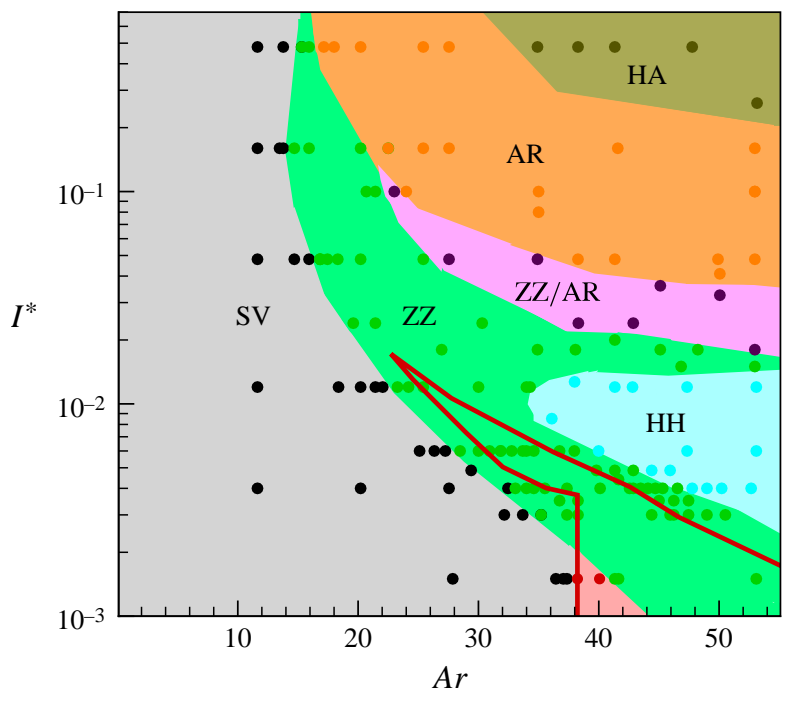

FIgURE 2. Phase diagram of the regimes of fall of an infinitely thin disk in the range $1.5 \times 10^{-3} \leqslant I^{*} \leqslant 5 \times 10^{-1}, A r \leqslant 55$. Black dots/grey zone: SV regime; green: ZZ regime; cyan: three-dimensional HH regime; purple: ZZ/AR regime; olive: three-dimensional HA regime; pink: small-amplitude planar zigzag similar to the $A$-regime (iii) in figure $\ulcorner(a)$. The red curve bounds the domain within which the various $A$-regimes are observed.

latter. All transitions are figured with sharp changes of colour but, based on the above discussion, it is likely that some of them actually correspond to a bistable behaviour. We did not examine this aspect in detail in the whole parameter space, since the $A r$ and $I^{*}$ steps we used to explore the dynamics of the system were often coarse, owing to limitations in the computational resources. Nevertheless we carefully considered the $\mathrm{SV}-\mathrm{ZZ}$ transition for low-inertia disks, which allowed us to determine the red line bounding the domain within which all aforementioned $A$-regimes are observed. This domain almost overlaps the lower part of the green region corresponding to the $\mathrm{ZZ}$ regime, except for the small triangular pink zone within which only the $A$-regime (iii) of figure $\Gamma(a)$ corresponding to a small-amplitude planar zigzag exists. Threedimensional $A$-regimes (i) and (ii) of figure $\Gamma(a)$ are only encountered in the upper 'horn' of the domain enclosed within the red line, whereas only the small-amplitude planar zigzag regime (iii) is present in the lower part of that domain, say $I^{*} \leqslant 3 \times 10^{-3}$.

According to this regime map, increasing $A r$ first makes the axial symmetry of the SV regime disappear, giving way to a planar symmetry and an average symmetry about the vertical axis that characterize the $\mathrm{ZZ}$ regime, as well as the small-amplitude zigzag observed for $I^{*} \leqslant 2 \times 10^{-3}$. The minimum $A r$ at which the $\mathrm{ZZ}$ path is first observed is detected for $I^{*}=0.16$ and corresponds to $A r \approx 14$, thus $\operatorname{Re}_{m} \approx 33$ (an ongoing global stability analysis of the disk + fluid system carried out in our team with a distinct code fully confirms this result). This threshold does not vary much for larger $I^{*}$; in contrast it sharply increases for lighter disks with $I^{*}<5 \times 10^{-2}$. Beyond the $\mathrm{ZZ}$ regime, two different symmetry breaking scenarios are observed, depending on the value of $I^{*}$. At low enough $I^{*}$, the planar symmetry is lost through the $\mathrm{ZZ}-\mathrm{HH}$ transition but the average vertical symmetry is preserved. Larger $I^{*}$ yield the opposite scenario since the ZZ-ZZ/AR transition preserves the planar symmetry but results in paths exhibiting a non-zero mean inclination. Then, during the tertiary 


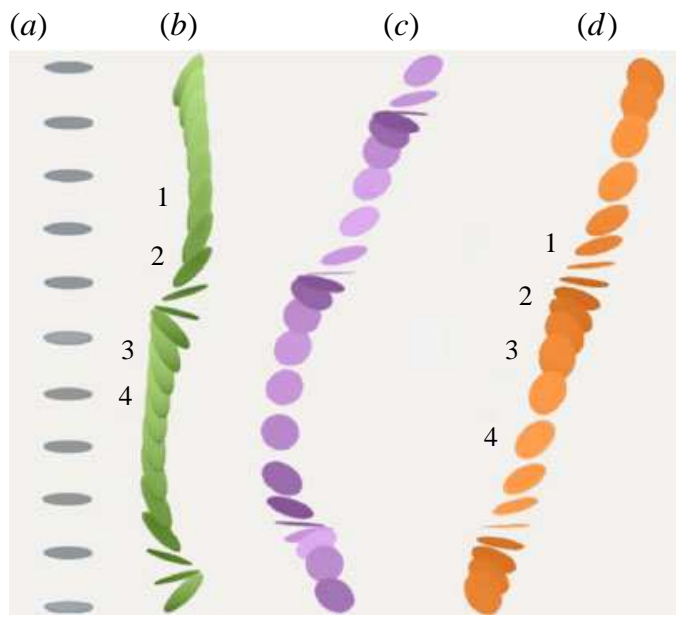

FIGURE 3. (Colour online) The four styles of planar paths: (a) SV $\left(A r=27.8, I^{*}=4 \times 10^{-3}\right)$; (b) $\mathrm{ZZ}\left(A r=25.5, I^{*}=4.8 \times 10^{-2}\right)$; (c) ZZ/AR $\left(A r=27.8, I^{*}=4.8 \times 10^{-2}\right)$; (d) AR $\left(A r=27.8, I^{*}=0.16\right)$. The lighting is proportional to the disk inclination; all four paths are strictly planar although the perspective effect may sometimes suggest that some threedimensionality is present, especially in case $(c)$.

AR-HA transition, the planar symmetry is lost while the average vertical symmetry is recovered.

As far as planar regimes are concerned, the above picture is qualitatively in line with the regime map reported by Field et al. ( $\square)$, except that the SV-ZZ transition was detected at strikingly larger Reynolds numbers there, typically $R e_{m} \gtrsim 90$ whatever $I^{*}$. In addition to finite- $\chi$ effects, the main reason for this difference certainly lies in the weak growth rates of the path instability close to the SV-ZZ threshold for disks with a significant inertia: in such cases we often noticed that the disk had to fall over distances of several hundreds of $d$ before reaching a saturated ZZ path. Since available laboratory observations were carried out in tanks whose height hardly exceeded some tens of $d$, it is unlikely that saturation was reached.

\subsection{Planar paths}

The four styles of planar paths we observe are illustrated in figure $\Gamma$ (see also supplementary movies). The corresponding diagrams based on the vertical $\left(U_{v}\right)$ and horizontal $\left(U_{h}\right)$ velocities in figure $\Gamma$ make the kinematic sequence in the periodic $\mathrm{ZZ}$ and $\mathrm{AR}$ paths and the chaotic nature of the ZZ/AR path explicit. Note that, although the Reynolds number is modest in these examples $\left(R e_{m} \lesssim 80\right)$, nonlinear effects are already strong: the maximum horizontal velocity is about half the mean vertical velocity in the $\mathrm{ZZ}$ regime $\left(R e_{m} \approx 64\right)$, while in the $\mathrm{AR}$ regime the ratio $U_{h} / U_{v}$ indicates that the disk's mean angle of descent $\gamma$ is $\sim 30^{\circ}\left(R e_{m} \approx 82\right)$, to be compared with $\gamma \approx 36^{\circ}$ with two-dimensional falling cards at $R e_{m} \approx 100$ according to Mahadevan, Ryu \& Samuel ( $\square$ ). The characteristics of these paths experience noticeable variations within their range of existence. Effects of $A r$ in the $\mathrm{ZZ}$ regime of light disks have already been discussed in connection with figure $\Gamma$. Heavier disks $\left(I^{*} \gtrsim 4.8 \times 10^{-2}\right)$ display a strikingly different behaviour: the amplitude of the $\mathrm{ZZ}$ path (in terms of $l_{h}$ and $\theta$ ) is very small when $A r$ is close to the SV-ZZ transition and strongly increases as $A r$ approaches the upper limit of existence of the regime, 

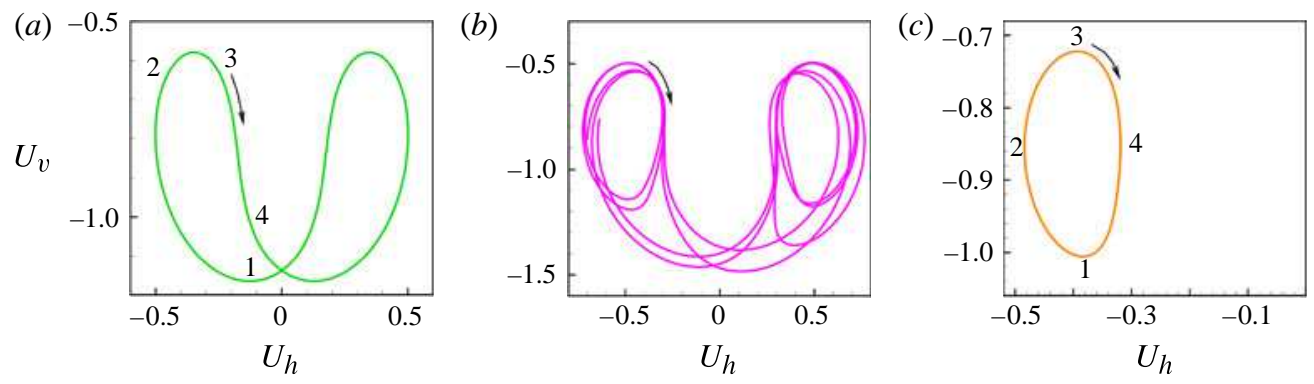

FIGURE 4. (Colour online) Velocity diagrams built on the vertical $\left(U_{v}\right)$ and horizontal $\left(U_{h}\right)$ velocities (both normalized with $U_{g}$ ) for the three time-dependent planar paths of figure $\Gamma:(a)$ periodic ZZ regime; $(b)$ chaotic ZZ/AR regime; $(c)$ periodic AR regime. The arrow indicates the direction followed by the disk. Labels 1-4 in $(a, c)$ refer to the same instants of time as in figure $\Gamma$. In both cases, $\left|U_{v}\right|$ is maximum at 1 where the disk glides on its side; $\left|U_{h}\right|$ is maximum at 2 where the disk inclination is minimum. This quickly leads to a strong drag increase resulting in a minimum of $\left|U_{v}\right|$ at 3 . At 4, the inertia of the disk is sufficient to allow it to overturn in $(c)$, resulting in a glide on its other side in the same horizontal direction, while in $(a)$ the fluid reaction dominates and reverses the direction of $U_{h}$, leading to a glide in the opposite direction.

where inclinations close to $90^{\circ}$ are encountered. Moreover, increasing $A r$ decreases the Strouhal number of disks with $I^{*} \gtrsim 2 \times 10^{-2}$, while it increases that of lighter disks. Despite these differences, $S t$ exhibits a regular variation with $I^{*}$ at the onset of the $\mathrm{ZZ}$ regime, where we find $S t \sim I^{*-0.22}$ (to be compared with $S t \sim I^{*-0.3}$ in Willmarth et al. ( $\square$ ) where the Strouhal number is based on $U_{m}$ rather than $U_{g}$ ): like in a classical oscillator, increasing inertia decreases the oscillation frequency.

In the range of existence of the AR regime, increasing $A r$ results in a slight increase of both the tumbling frequency (hence $S t$ ) and $\gamma$. Within the chaotic ZZ/AR regime, these influences may combine in such a way that during some stages the disk temporarily ascends, as we observed for $I^{*} \approx 2 \times 10^{-2}$ by increasing $A r$ up to 70; such a behaviour has already been reported with almost two-dimensional falling cards (Andersen, Pesavento \& Wang $\square$ ). Varying $I^{*}$ makes $S t$ vary like $S t \sim I^{*-0.36}$ at the onset of the regime (to be compared with $S t \sim I^{*-1 / 2}$ in the fully developed tumbling regime of two-dimensional falling cards (Mahadevan et al. $\square$ )). Since a tumbling period is made of a glide followed by a rotation while both stages repeat twice during a period of zigzag, one expects the frequency of the former style of path to be typically twice that of the latter when both exist for a given $I^{*}$. Observations confirm this reasoning with, for instance for $I^{*}=4.8 \times 10^{-2}, S t \approx 0.31$ in the AR regime for $A r \approx 32$ and $S t \approx 0.15$ in the $\mathrm{ZZ}$ regime for $A r \approx 26$. Still in the $\mathrm{AR}$ regime, heavy disks (such as that of figure $\ulcorner d$ ) tend to follow an almost straight path, while lighter ones display a more undulated trajectory, their smaller inertia resulting in a larger sensitivity to unsteady aerodynamic loads. Close to the lowest frontier of that regime $\left(I^{*} \approx 2 \times 10^{-2}, A r \geqslant 50\right)$, the path is found to exhibit a subharmonic modulation, yielding a cycle consisting of a succession of 'short' and 'long' glides, as already noticed with two-dimensional cards (Andersen et al. $\square$ ).

\subsection{Three-dimensional paths}

Compared to experimental data (Willmarth et al. $\square$; Stringham et al. $\square$; Field et al. $\square$, the novel feature observed here for large enough $A r$ is the occurrence 

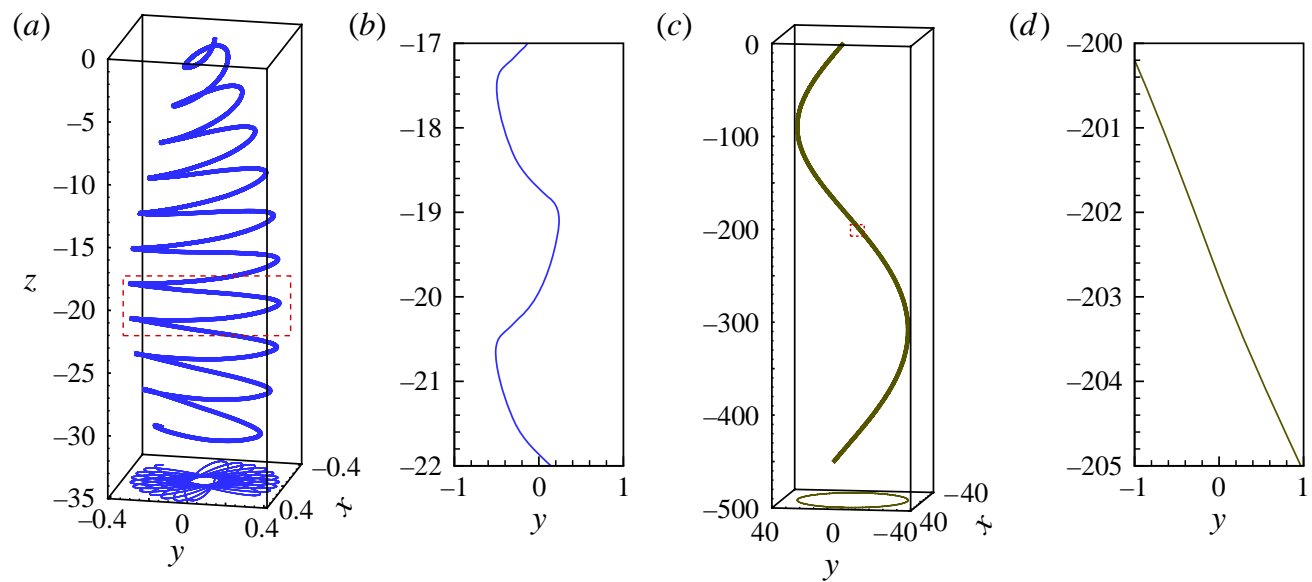

FIgURE 5. (Colour online) The two three-dimensional styles of path: $(a) \mathrm{HH}$ path for $A r=47.95, I^{*}=4 \times 10^{-3}\left(R e_{m} \approx 124\right) ;(c)$ HA path for $A r=47.9, I^{*}=0.48\left(R e_{m} \approx 133\right)$. Note that the vertical scale is strongly compressed in both plots. (b) (respectively $d$ ): zoom of (a) (respectively $c$ ) during a stage when the path lies approximately in the $(y, z)$ plane.
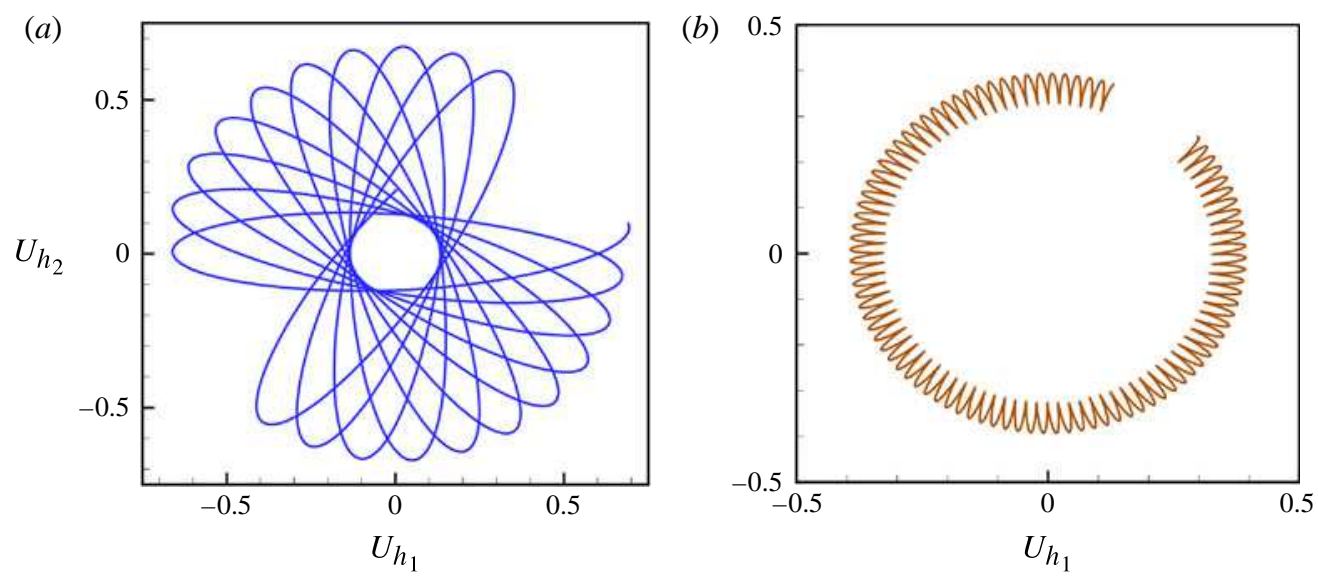

FIGURE 6. (Colour online) Velocity diagrams built on horizontal velocity components (both normalized with $U_{g}$ ) in two arbitrary perpendicular directions for the two three-dimensional paths displayed in figure $\digamma$ : $(a) \mathrm{HH}$ regime; $(b)$ HA regime.

within two ranges of $I^{*}$ of fully three-dimensional paths (figure $\Gamma$ ) characterized by well-defined periodic horizontal projections (figure $\ulcorner$ ) (see also supplementary movies). This is the case of light disks in the range $3 \times 10^{-3}<I^{*}<2 \times 10^{-2}, A r \gtrsim 45$ which exhibit a clear $\mathrm{HH}$ path. This is also the case for sufficiently heavy disks which, for $A r \gtrsim 30$, may switch from an AR path to a three-dimensional HA path. Again, the plane in which the tumbling motion takes place slowly rotates (by $\sim 4^{\circ}$ per period of tumbling in figures $\Gamma c$ and $\Gamma b$ ), making the disk centre of mass slightly wobble around a circular helix with a large diameter $(\sim 30 d$ in the above example). The pitch of these three-dimensional paths is very large (e.g. the disk falls by $\sim 65 d$ and $440 d$ during a complete rotation in the $\mathrm{HH}$ and $\mathrm{HA}$ examples of figure $\Gamma$, respectively), 
which again explains why they have not yet been detected in experiments (in the case of the HA regime, another reason is that sufficient values of $I^{*}$ cannot be reached in water. However, they may certainly be obtained for instance by dropping small disks $(d \approx 2 \mathrm{~cm})$ made of Bristol board $\left(\sigma_{s} \approx 0.2 \mathrm{~kg} \mathrm{~m}^{-2}\right)$ in air). Owing to this weak change in the path geometry, no significant frequency jump is noticed across the $\mathrm{ZZ}-\mathrm{HH}$ and $\mathrm{AR}-\mathrm{HA}$ transitions, in contrast to what happens across the $\mathrm{ZZ}-\mathrm{AR}$ transition.

The so-called 'transitional' regime recently observed by Zhong et al. ( $\square)$ with disks of very low inertia $\left(7 \times 10^{-4} \leqslant I^{*} \leqslant 3 \times 10^{-3}\right)$ exhibits paths reminiscent of the $\mathrm{HH}$ style. However, the corresponding pitch is much smaller, as the disk falls by only 3-4d while performing a complete revolution in the horizontal plane. Moreover, for a given $I^{*}$, this style of path was found to exist in a range of $A r$ intermediate between those corresponding to the $\mathrm{SV}$ and $\mathrm{ZZ}$ regimes, i.e. the observed transition sequence as $\mathrm{Ar}$ increases is $\mathrm{SV}$-transitional-ZZ, whereas the $\mathrm{HH}$ regime we detect at slightly larger $I^{*}$ belongs to a SV-ZZ-HH scenario. This is why, although they exhibit qualitatively similar path geometries, we think that the present $\mathrm{HH}$ regime differs from the 'transitional' regime reported by Zhong et al. ( $\square)$. We also note that the same authors pointed out the existence of a 'spiral' mode in which the disk falls along a straight vertical path while rotating with a constant non-zero inclination. We did not observe this mode, which is consistent with the fact that it was detected only for $I^{*} \leqslant 7.5 \times 10^{-4}$ in the experiments, a range of inertia ratios which is below the lower $I^{*}$-limit of the domain we explored.

\section{Wakes versus paths}

\subsection{Structural differences between wake instability of a fixed disk and instability of the coupled fluid + disk system}

The presence of vorticity in the flow is entirely responsible for the onset of path instability for disks and other axisymmetric bodies released with their symmetry axis aligned vertically, as well as for two-dimensional cards released broadside on. Therefore, one intuitively expects the bifurcations encountered for such a falling body to closely mirror those occurring in the wake of the same body, when held fixed in a uniform stream, as if the body path were slaved to the wake. This expectation turns out to be essentially wrong. First, the threshold at which the first bifurcation occurs may be extremely different in the two problems. For instance, for an infinitely thin disk, the first bifurcation of the flow in the fixed-body problem (which leads to a steady, non-axisymmetric wake) occurs for $R e=115.5$ (corresponding to $A r \approx 38.5$ if the disk were free to fall), a value much larger than the threshold of the SV-ZZ transition over most of the range of $I^{*}$ explored here. A similar trend has been reported by Alben ( $\square$ ) with two-dimensional elliptical bodies: the critical Reynolds number beyond which the body assigned to rise with a fixed velocity but free to move horizontally exhibits a non-zero lateral motion was observed to be nearly half that corresponding to the threshold of the instability of the fixed-body wake. As we saw, the bifurcation threshold in the fixed-body problem only mildly depends on $\chi$, reaching $R e=127$ for $\chi=10$ (corresponding to $A r \approx 41.8$ if the disk were free to fall), in sharp contrast with the results presented above. Then, the bifurcation scenario for fixed disks with $\chi \geqslant 10$ which, up to $R e=185$, involves a succession of two periodic regimes with different symmetries (Fabre, Auguste \& Magnaudet $\square$ ) also totally differs from the various SV-ZZ transition scenarios encountered here. Last but not least, the frequency of the periodic fluttering in the $\mathrm{ZZ}$ regime generally 
differs much from the vortex shedding frequency observed with fixed disks. This is particularly striking for light disks: as we saw with $I^{*}=4 \times 10^{-3}$, the Strouhal number at the onset of the $\mathrm{ZZ}$ regime is 0.235 , i.e. 0.29 when normalized by $U_{m}$ instead of $U_{g}$, to be compared with $S t \approx 0.12$ for a fixed disk. Thus, as already noticed with rising oblate bubbles (Mougin \& Magnaudet $\square$ ) and falling two-dimensional cards (Assemat et al. $\square$ ), one has to conclude that the wake dynamics are fully coupled to the movements of the disk (actually to its accelerations), even though the amplitudes of the latter are modest. The connection between the dynamics of the fully coupled system and that of the fixed-body wake was examined in detail in the latter reference (see also Fabre, Assemat \& Magnaudet $\square$ ) using linear stability. This analysis revealed existence of several unstable modes for a given $I^{*}$, some of which (termed as 'aerodynamic' in the limit of large $I^{*}$ by Fabre et al.) result in significant body oscillations for a given energy of the mode, while others (termed as 'fluid' in the same limit) essentially induce fluctuations in the fluid without significantly affecting the body motion. Shedding frequencies similar to the dominant frequency of the fixed-body wake are observed when the most unstable mode belongs to the latter type, while fluttering or tumbling frequencies totally different from this 'fixed-body' frequency emerge with the other family of modes.

\subsection{Physical mechanisms governing the style of path}

The role of the above couplings is crucial for sustaining significant transverse motions of the disk and selecting its style of path. In particular, the rotation of the disk about one of its diameters generates variations in the fluid velocity distribution along the edge, resulting in larger vertical fluid velocities along the descending part when the disk inclination is small. Owing to the vortex tilting mechanism, this in turn drives the generation of a significant streamwise vorticity component which progressively forms a hairpin vortex that elongates in the downstream direction as time elapses. When this vortex is released downstream, there is a period of time during which the wake-induced torque is almost zero and the torque balance is momentarily controlled by purely inertial effects associated with the disk + fluid system, as if the flow were essentially irrotational (Lamb $\square$ ). Then, depending on the disk-to-fluid relative inertia, two main scenarios may take place for large enough $A r$. If $I^{*}$ is low enough, fluid inertia is dominant during that stage and the torque balance is controlled by the added-mass contribution which tends to realign the disk axis with its instantaneous velocity. In so doing, it forces the disk transverse velocity to vanish, yielding the zigzag style of path and making the cycle start again with a reversed sign of the disk rotation and thus of the streamwise vorticity in each arm of the hairpin (figure $\ulcorner a-d$ ).

In contrast, if $I^{*}$ is large enough, significant non-zero values of the added-mass torque can be balanced by the disk's rotational acceleration during that stage. Thus, the disk axis and its velocity may stay misaligned during some time without inducing any sign reversal in the disk rotation. Provided viscous effects are small enough, this allows the disk inclination to keep on increasing, eventually yielding complete overturning. However, at large inclination, the fluid velocity distribution along the edge is not primarily controlled by rotation any more but rather by the fore-aft flow asymmetry which results in larger fluid velocities near the bottom part of the edge. Combining the effect of this asymmetry for large inclinations with that of rotation for small inclinations, it is easy to figure out that the point of the edge facing the largest fluid velocity when the disk is vertical faces the lowest fluid velocity when it gets horizontal, and vice versa. This is why two successive hairpin vortices with opposite sign are encountered during a tumbling half-period: one starts growing before 
(a)

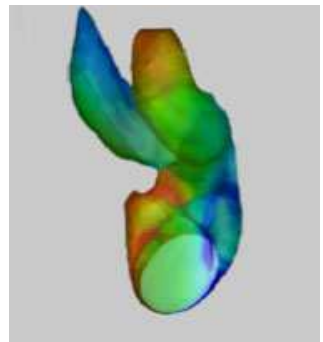

(b)

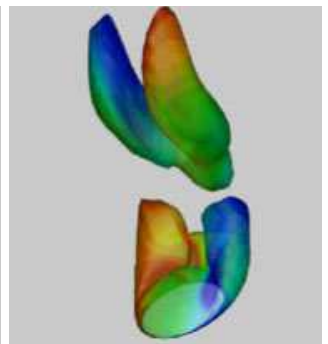

(c)

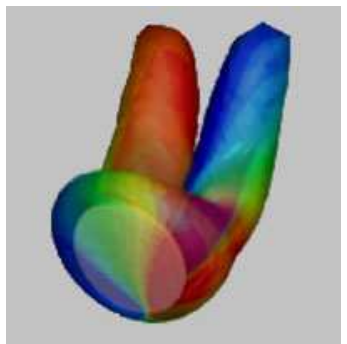

$(d)$

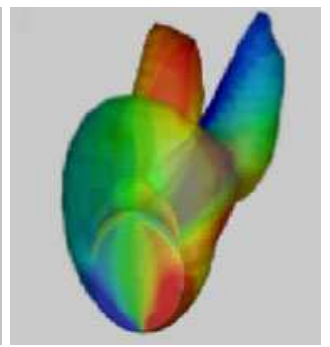

FIGURE 7. Evolution of the hairpin vortex structures during a zigzag half-period $(A r=25.5$, $\left.I^{*}=4.8 \times 10^{-2}\right) ;(a-d)$ refer to labels $1-4$ in figure $\Gamma(b)$. The wake is visualized using the $\lambda_{2}$ criterion (Jeong \& Hussain $\square$ ); the colour scale on the $\lambda_{2}$ iso-surface refers to the local value of the vertical vorticity. The clockwise rotation of the disk is accompanied by the formation of a pair of streamwise vortices; in $(a-b)$, a similar structure of opposite sign formed during the previous half-period is visible in the upper part of the wake. See also supplementary movies.

(a)

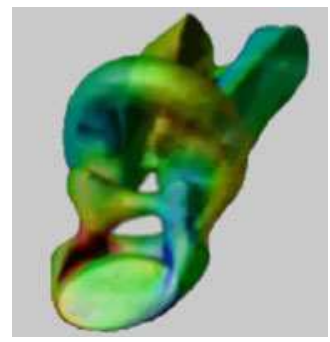

(b)

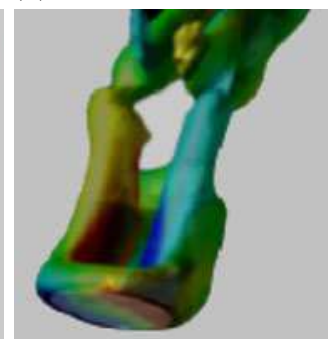

(c)

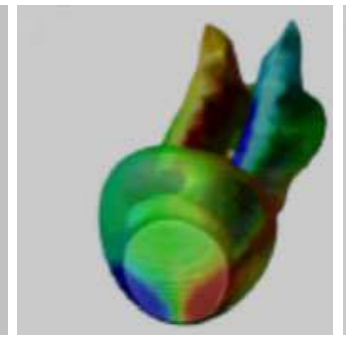

$(d)$

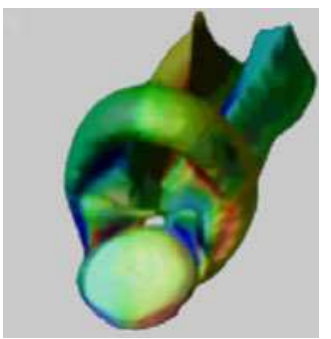

FIGURE 8. Evolution of the hairpin vortex structures during a tumbling sequence $(A r=27.8$, $\left.I^{*}=0.16\right) ;(a-d)$ refer to labels $1-4$ in figure $\Gamma(d)$. See figure $\ulcorner$ for legend. Two threads of streamwise vorticity form behind the bottom part of the edge of the disk when it is nearly vertical in $(c)$; this yields a hairpin vortex whose central part detaches with a topdown asymmetry whilst a second hairpin vortex of opposite sign starts forming just behind the disk in $(d)$; the former structure is completely released in the wake whilst the latter is growing in $(a)$ where the disk is nearly horizontal; this second vortex strongly elongates in $(b)$, and eventually detaches when the disk is back in $(c)$ with its two faces inverted. See also supplementary movies.

the plane of the disk becomes vertical (figure $\lceil c$ ), then elongates (figure $\lceil d$ ) and is released downstream when the disk inclination is close to $30^{\circ}$ (figure $\Gamma a$ ). The hairpin vortex of opposite sign already started growing (figure $\ulcorner d$ ), before elongating (figure $\lceil a$ ) and being released downstream some time before the disk gets back to vertical. Obviously, the constant sign of the disk rotation results in an asymmetric shedding of the vortices with respect to the vertical (figure $\Gamma a, d$ ), yielding the mean horizontal drift of the disk typical of the AR regime.

Path symmetries of course mirror wake symmetries and vice versa. Hence, planar paths are associated with wake geometries preserving a reflectional symmetry (figure $\lceil a$ ). However, this symmetry may break when the strength of viscous effects falls below some critical threshold, then inducing a permanent drift of the body 
(a)

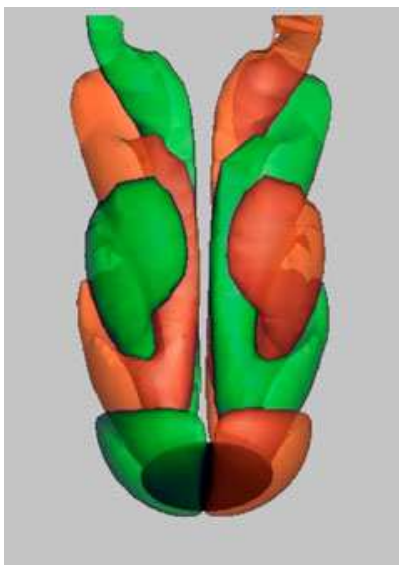

(b)

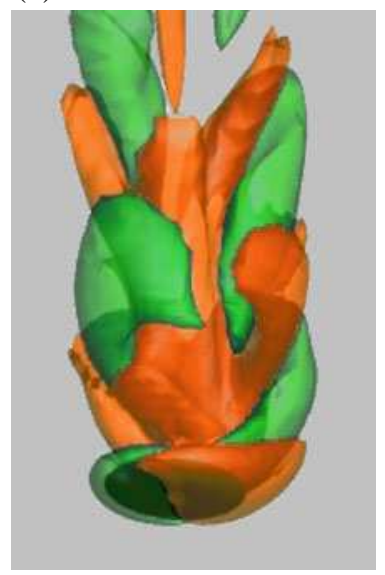

FIGURE 9. Geometry of the streamwise vorticity iso-surfaces in the wake of a disk with: $(a)$ $I^{*}=4 \times 10^{-3}$ and $A r=33.2\left(R_{m} \approx 90\right)$ in the $\mathrm{ZZ}$ regime; $(b) I^{*}=4 \times 10^{-3}$ and $A r=47.9$ $\left(R e_{m} \approx 124\right)$ in the $\mathrm{HH}$ regime.

path, as already demonstrated with simple two-dimensional oscillating systems, both experimentally (Vandenberghe, Zhang \& Childress $\square$ ) and numerically (Alben \& Shelley $\square$ ). Here this symmetry loss results in a twisted wake structure (figure $\ulcorner b$ ) which induces a mean self-rotation of the disk, resulting in a slow drift of its plane of fall. This is how, starting from the planar ZZ (respectively, AR) regime, the system bifurcates within some range of $I^{*}$ toward the three-dimensional HH (respectively, HA) regime beyond a critical $A r$.

\section{Conclusions}

We have reported on results obtained during what seems to be the first systematic computational study of the dynamics of thin rigid disks falling in a Newtonian fluid at rest at infinity. Compared with available experiments, our study is restricted to low-to-moderate Reynolds numbers (typically $R e_{m} \lesssim 200$ for $\chi=\infty$ ) but extends over a wide range of solid-to-fluid inertia ratios $\left(1.5 \times 10^{-3} \leqslant I^{*} \leqslant 0.5\right)$ and includes some situations with high $I^{*}$ that cannot be studied in water tanks. By considering disks with a finite, albeit large, aspect ratio $(\chi \geqslant 10)$ as well as infinitely thin disks $(\chi=\infty)$, present results show that, at variance with previous beliefs, the dynamics of the system strongly depend on $\chi$ : both the transition sequence and the thresholds of the non-straight paths reveal a crucial influence of $\chi$ for aspect ratios of some tens, questioning available regime maps which mix results obtained with disks of various aspect ratios. The transition to the zigzagging path also revealed existence of a series of intermediate regimes characterized by small path deviations, and of regions of the parameter space where the system exhibits a clear bistability. Therefore, the actual route from the straight vertical path to a well-defined zigzagging regime is far from reducing to a single bifurcation.

In the presence of significant deviations from the straight vertical path, five distinct dynamical regimes have been observed with infinitely thin disks. Three of them correspond to the already documented planar paths, with two periodic styles of motion, zigzagging (三fluttering) and autorotation (三tumbling), separated up to $I^{*} \approx 10^{-1}$ by 
a chaotic regime which combines both of them. As viscous effects become small enough by increasing the Archimedes number, the planar symmetry of the wake and path may be broken, yielding twisted wake structures and three-dimensional paths in which the plane of the fluttering/tumbling motion slowly rotates about the vertical axis. These styles of path require a long time to develop, which is certainly the reason why they have not been reported before, owing to the limited height of observation tanks. The characteristics of the observed dynamics make it clear that, in a given range of Reynolds number, wakes behind freely falling and fixed disks are strikingly different from each other, even in presence of small accelerations. These couplings drive the generation and shedding of hairpin vortices in the wake, which, together with the relative magnitude of inertial effects associated with the body and surrounding fluid, govern the disk's style of path.

Extensive computations are now required to explore the detailed dynamics of the various transitions (especially the nature of the higher-order bifurcations that was not discussed here), consider lower $I^{*}$ for which the sensitivity of the response of the disk to the fluid is expected to be even greater than in the range considered here, and go toward higher $\mathrm{Ar}$ for which all paths probably become three-dimensional.

\section{Acknowledgement}

Part of this work was supported by the Agence Nationale de la Recherche under grant ANR-09-BLAN-0132 OBLIC.

\section{Supplementary movies}

Supplementary movies are available at http://dx.doi.org/10.1017/jfm.2012.602.

\section{Appendix A. Grid, boundary conditions and validation tests}

In this appendix we provide details regarding various aspects of the grid characteristics, boundary condition on the outer surface of the numerical domain and validation tests of the whole approach.

Regarding boundary conditions, a no-slip condition is of course imposed at the disk surface, while a specially designed condition is applied on the whole outer surface of the domain to minimize wake-induced disturbances. This condition, which evolves in time with the orientation of the wake, is based on the sign of the normal velocity disturbance, $V_{n}$, detected at each time step in each grid cell located along the outer boundary of the domain. The velocity disturbance is set to zero on parts of this boundary on which $V_{n}$ is found to be positive (the flow is almost irrotational in these regions, implying that the corresponding disturbance is very small, as it decays like $r^{-3}$ for large distances $r$ to the body centre of mass). In contrast, following Magnaudet, Rivero \& Fabre ( $\square$ ), the first-order (respectively, second-order) normal derivative of the tangential (respectively, normal) velocity disturbance is set to zero together with the second-order cross-derivative of the pressure disturbance on the parts of the boundary characterized by negative values of $V_{n}$, i.e. intersecting the wake. Extensive tests (Auguste $\square$ ) showed that, compared with standard zero-velocity or stress-free far-field conditions, this time-evolving condition drastically reduces the influence of the outer boundary on the dynamics of the system, allowing for the use of significantly smaller computational domains. In particular, compared with the above two standard conditions, it was found to provide negligible pressure fluctuations in the vicinity of a disk forced to describe complete end-over-end flips at various frequencies. 
A strictly infinitely thin disk $(\chi=\infty)$ is obtained by imposing a no-slip condition on both sides of the common frontier between two successive grid slices. The presence of a sharp edge where the exact solution of the flow problem is singular requires specific attention to make sure that the discrete velocity and pressure fields near the edge convey the proper characteristics of this exact solution and that the spreading of the singularity due to the finite discretization does not affect the overall dynamical quantities, especially the force and torque experienced by the body. We carried out extensive tests throughout the range of Reynolds number of interest here and concluded that the above requirements are met in all cases when the size of the grid cells is of $O\left(10^{-2} d\right)$ near the edge. We specifically checked that, in the absence of significant effects of fluid inertia, the features of the singular theoretical solution (Moffat $\square$ ) are quantitatively recovered close to the edge, and that computational results agree well with theoretical solutions describing the flow about a disk undergoing arbitrary translations and rotations in a quiescent fluid (Tanzosh \& Stone $\square$ ). As fluid inertia increases, viscous effects near the disk surface concentrate within a boundary layer whose thickness is expected to decrease to $\sim 0.06 d$ for the largest Reynolds numbers under consideration. Since the present code is known to properly capture viscous effects at moderate-to-large Reynolds numbers when at least three grid points lie within the boundary layer (Blanco \& Magnaudet $\square$ ), the above resolution is also appropriate in the direction normal to the disk surface. Finally, viscous effects in the near wake must also be properly captured. We found that this is achieved by requesting the cell size to be less than $0.1 d$ at all grid points located within a $3 d$ distance from the disk centre. In summary, within this central region of the computational domain, the spatial resolution of the grids with which the results discussed above were obtained varies gradually from $O\left(10^{-2} d\right)$ to $0.1 d$ in the longitudinal direction as the distance to the disk increases; in the radial direction it first decreases from $O\left(5 \times 10^{-2} d\right)$ on the axis of the disk to $O\left(10^{-2} d\right)$ near its edge and then increases gradually up to $0.1 d$. With this discretization, the first bifurcation in the wake of a fixed disk whose axis is aligned with the upstream flow is found to occur at $R e=115.5$, a threshold which differs by less than $1 \%$ from that determined by Natarajan \& Acrivos ( $\square$ ) using a global linear stability approach. The extensive tests we carried out to determine the influence of the spatial discretization in each direction with a disk held fixed in a uniform stream at $R e=100$ may be found in ch. 3 of Auguste $(\square)$; comparisons with the aforementioned low-Reynolds-number solutions may be found in appendix A of the same reference.

In addition to viscous effects, it is also crucial that the discrete solution properly captures the instantaneous changes induced in the flow field by an acceleration of the disk, i.e. the so-called added-mass effects. The added-mass coefficients corresponding to an arbitrary translational (respectively, rotational) acceleration can easily be computed using the short-time variations of the force (respectively, torque) experienced by the body in presence of an imposed acceleration of the upstream flow, following the procedure described by Rivero, Magnaudet \& Fabre ( $\square$ ) (see also Mougin \& Magnaudet $\square$ ). We used this procedure to compare the computed values of the longitudinal and transverse added-mass coefficients for disks of various aspect ratios with the theoretical solutions provided by Loewenberg $(\square)$. The agreement was found to be better than $2 \%$ for all $\chi$, which in particular indicates that the above discretization allows the flow to properly circumvent the edge in presence of a differential acceleration between the body and fluid. The body displacements are obtained by integrating the Kirchhoff-Kelvin equations twice in time using a thirdorder Runge-Kutta scheme. Hence, the minimum lateral displacement $\delta_{m}$ of the disk 
that can be captured by the numerical scheme is proportional to the square of the time step, which, owing to the Courant-Friedrichs-Lewy stability condition, evolves linearly with the minimum cell size $\Delta_{m}$. Therefore, it is a simple matter to show that for a disk whose axis is inclined by a small angle $\gamma$ with respect to the vertical, having set $\Delta_{m}$ to $O\left(10^{-2} d\right)$ yields $\delta_{m} / d=O\left(10^{-4} \gamma\right)$.

Using the outer boundary condition and features of the spatial discretization described above, the various series of tests we carried out allowed us to determine that a $10 \mathrm{~d}$ radius, $20 \mathrm{~d}$ long cylindrical domain discretized with a grid made of $100 \times 80 \times 32$ cells in the streamwise, radial and azimuthal directions, respectively, provides grid-independent results within the desired range of Reynolds or Archimedes number. In particular, we checked that doubling the spatial resolution near the edge or the number of azimuthal planes did not produce any discernible change in the path of a freely moving disk under various conditions. In the inertia-dominated regimes of interest here, extensive comparisons with recent experiments (Fernandes et al. $\square$ ) were carried out for aspect ratios $\chi=1,3,10$ and revealed excellent agreement, especially on the thresholds, amplitudes and frequencies of the oscillatory motions (see ch. 8 of Auguste $(\square)$ ).

\section{REFERENCES}

Alben, S. 2008 An implicit method for coupled flow-body dynamics. J. Comput. Phys. 227, 4912-4933.

Alben, S. \& Shelley, M. 2005 Coherent locomotion as an attracting state for a free flapping body. Proc. Natl Acad. Sci. U.S.A. 102, 1163-1166.

Andersen, A., Pesavento, U. \& Wang, Z. J. 2005 Unsteady aerodynamics of fluttering and tumbling plates. J. Fluid Mech. 541, 65-90.

Assemat, P., Fabre, D. \& Magnaudet, J. 2012 The onset of unsteadiness of two-dimensional bodies falling or rising freely in a viscous fluid: a linear study. J. Fluid Mech. 690, 173-202.

Auguste, F. 2010 Instabilités de sillage et trajectoires d'un corps solide cylindrique immergé dans un fluide visqueux. PhD thesis, Université Paul Sabatier, Toulouse, France, available at https://www.imft.fr/Projet-ANR-OBLIC.

Blanco, A. \& Magnaudet, J. 1995 The structure of the axisymmetric high-Reynolds number flow around an ellipsoidal bubble of fixed shape. Phys. Fluids 7, 1265-1274.

Chrust, M. 2012 Etude numérique de la chute libre d'objets axisymétriques dans un fluide newtonien. PhD thesis, Université de Strasbourg, France.

ERn, P., Risso, F., Fabre, D. \& Magnaudet, J. 2012 Wake-induced oscillatory paths of bodies freely rising or falling in fluids. Annu. Rev. Fluid Mech. 44, 97-121.

Fabre, D., Assemat, P. \& Magnaudet, J. 2011 A quasi-static approach to the stability of the path of heavy bodies falling within a viscous fluid. J. Fluids Struct. 27, 758-767.

Fabre, D., Auguste, F. \& Magnaudet, J. 2008 Bifurcation and symmetry breaking in the wake of axisymmetric bodies. Phys. Fluids 20, 051702.

Fabre, D., Tchoufag, J. \& Magnaudet, J. 2012 The steady oblique path of buoyancy-driven disks and spheres. J. Fluid Mech. 707, 24-36.

Fernandes, P. C., Ern, P., Risso, F. \& Magnaudet, J. 2005 On the zigzag dynamics of freely moving axisymmetric bodies. Phys. Fluids 17, 098107.

Fernandes, P. C., Ern, P., Risso, F. \& Magnaudet, J. 2007 Dynamics of axisymmetric bodies rising along a zigzag path. J. Fluid Mech. 606, 209-223.

Field, S., Klaus, M., Moore, M. \& Nori, F. 1997 Chaotic dynamics of falling disks. Nature 388, 252-254.

Jeong, J. \& Hussain, F. 1995 On the identification of a vortex. J. Fluid Mech. 285, 69-94.

LAMB, SIR H. 1932 Hydrodynamics. Cambridge University Press.

Loewenberg, M. 1993 Stokes resistance, added mass and Basset force for arbitrarily oriented, finite-length cylinders. Phys. Fluids A5, 765-767. 
Magnaudet, J., Rivero, M. \& Fabre, J. 1995 Accelerated flows past a rigid sphere or a spherical bubble. Part 1. Steady straining flow. J. Fluid Mech. 284, 97-135.

Mahadevan, L., Ryu, W. S. \& Samuel, D. T. 1999 Tumbling cards. Phys. Fluids 11, 1-3.

Moffat, H. K. 1964 Viscous and resistive eddies near a sharp corner. J. Fluid Mech. 18, 1-18.

Mougin, G. \& Magnaudet, J. 2002a The generalized Kirchhoff equations and their application to the interaction between a rigid body and an arbitrary time-dependent viscous flow. Intl $J$. Multiphase Flow 28, 1837-1851.

Mougin, G. \& Magnaudet, J. $2002 b$ Path instability of a rising bubble. Phys. Rev. Lett. 88, 14502.

Mougin, G. \& Magnaudet, J. 2006 Forces and torques on a zigzagging/spiralling bubble. J. Fluid Mech. 567, 185-194.

NAtARAJAN, R. \& ACRivos, A. 1993 The instability of the steady flow past spheres and disks. J. Fluid Mech. 254, 323-344.

Rivero, M., Magnaudet, J. \& Fabre, J. 1991 New results on the force exerted on a spherical body by an accelerating flow. C. R. Acad. Sci. Paris Sér. II 312, 1499-1506.

Stringham, G. E., Simons, D. B. \& GuY, H. P. 1969 The behavior of large particles falling in quiescent liquids. US Geol. Surv. Prof. Pap. 562C, 1-36.

TAnzosh, J. P. \& Stone, H. A. 1996 A general approach for analyzing the arbitrary motion or a circular disk in a Stokes flow. Chem. Engng Commun. 150, 333-346.

VAndenberghe, N., Zhang, J. \& Childress, S. 2004 Symmetry breaking leads to forward flapping flight. J. Fluid Mech. 506, 147-155.

Willmarth, W., Hawk, N. \& Harvey, R. 1964 Steady and unsteady motions and wakes of freely falling disks. Phys. Fluids 7, 197-208.

Zhong, H., Chen, S. \& LEE, C. 2011 Experimental study of freely falling thin disks: transition from planar zigzag to spiral. Phys. Fluids 23, 011702. 